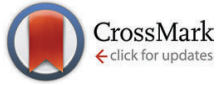

Cite this: J. Mater. Chem. C, 2016, 4, 3628

Received 30th December 2015, Accepted 4th April 2016

DOI: $10.1039 / \mathrm{c} 5 \mathrm{tc} 04448 \mathrm{k}$

www.rsc.org/MaterialsC

\section{Nanocrystals of $\mathrm{CuCr}_{2} \mathrm{~S}_{4-x} \mathrm{Se}_{x}$ chalcospinels with tunable magnetic properties $\dagger$}

\author{
Karthik Ramasamy, ${ }^{* a}$ Hunter Sims, ${ }^{b}$ Sahar Keshavarz, ${ }^{c}$ Nariman Naghibolashrafi ${ }^{c}$ \\ and Arunava Gupta*c
}

Ferromagnetic materials exhibiting large spin polarization at room temperature have been actively pursued in recent years for the development of next-generation spintronic devices. Chromium-based chalcospinels are the only ternary chalcogenide-containing magnetic materials with Curie temperatures above room temperature. However, the magnetic and electronic properties of chromium-based chalcospinels at the nanoscale level are not well understood. We have developed a facile colloidal method for the synthesis of $\mathrm{CuCr}_{2} \mathrm{~S}_{4-x} \mathrm{Se}_{x}(\mathrm{O} \leq x \leq 4)$ nanocrystals over the entire composition range. Systematic changes in the lattice parameter and elemental composition confirm formation of $\mathrm{CuCr}_{2} \mathrm{~S}_{4-x} \mathrm{Se}_{x}(0 \leq x \leq 4)$ nanocrystals. The dimensions of the nanocrystals, as determined from TEM images, vary from $12 \pm 1.4 \mathrm{~nm}$ to $21 \pm 1.4 \mathrm{~nm}$. The Curie temperature $\left(T_{C}\right)$ shows a systematic increase with increasing selenium content. Saturation magnetization and coercivity values of $\mathrm{CuCr}_{2} \mathrm{~S}_{4-x} \mathrm{Se}_{x}(0 \leq x \leq 4)$ nanocrystals at $5 \mathrm{~K}$ are found to steadily increase up to $x=3$. Electronic structure calculations as a function of composition and size using density functional theory suggest ferromagnetic ordering over the entire composition range with partial spin polarization for the bulk materials. Furthermore, the calculations predict complete opening-up of a gap at the Fermi level in the minority spin channel at reduced dimensions to render them completely spin polarized, i.e., display half-metallic characteristics.

\section{Introduction}

Spin-based devices offer a significant advantage over charge-based ones because they are typically nonvolatile, requiring zero idle power. ${ }^{1,2}$ This promises significant energy savings over purely charge-based electronics, allowing device density to be increased beyond limits imposed by manipulating charge alone. ${ }^{3}$ Present-day spintronic devices primarily utilize the tunneling magnetoresistance (TMR) effect, which is based on tunnel junctions consisting of two ferromagnetic electrodes separated by an insulating tunneling layer with thickness of only a few nanometers. ${ }^{4}$ The performance of TMR and other spintronic devices depends critically on the degree of spin polarization $(P)$ of the ferromagnetic electrodes. Of particular interest are the so-called half-metallic ferromagnets, which are completely spin polarized with $P=1 .^{5-7}$ A half-metallic material is simultaneously an excellent metal for majority-spin electrons and an insulator for

\footnotetext{
${ }^{a}$ Center for Integrated Nanotechnologies, Los Alamos National Laboratory, Albuquerque, NM-87185, USA.E-mail: kramasamy@lanl.gov

${ }^{b}$ German Research School for Simulation Sciences, Jülich, Germany-52425

${ }^{c}$ Center for Materials for Information Technology, The University of Alabama,

Tuscaloosa, AL-35487, USA. E-mail: agupta@mint.ua.edu

$\dagger$ Electronic supplementary information (ESI) available: Experimental and simulated XRD patterns for the different compositions, EDX elemental mapping, and $M-H$ data at $300 \mathrm{~K}$. See DOI: $10.1039 / \mathrm{c} 5 \mathrm{tc} 04448 \mathrm{k}$
}

minority-spin electrons. Because of selective spin injection, half-metallic materials can in principle be used as an ideal spin injector and detector in spin-logic devices. ${ }^{8}$ In a magnetic tunnel junction with half-metallic electrodes, one would have the exciting prospect of switching between conducting and insulating states when the relative magnetization orientations of the two electrodes are altered. Nevertheless, it is necessary that such ferromagnetic materials exhibit high spin polarization at or above room temperature for practical applications. For this purpose, chromium-based chalcogenide spinels are an attractive class of material possessing a wide variety of electronic and magnetic properties. ${ }^{9-14}$ In these materials, depending on A-site substitution, ferro/ferrimagnetic ordering with insulating/ semiconducting or metallic type transport properties can be realized. ${ }^{15}$ Electronic structure calculations predict high spin polarizations in a large number of chromium-based chalcospinel compounds, including some with complete half-metallic characteristics. ${ }^{16,17}$ Moreover, copper-containing chalcospinel ferromagnets such as $\mathrm{CuCr}_{2} \mathrm{E}_{4}(\mathrm{E}=\mathrm{S}$, Se or Te) meet the high Curie temperature requirement. The Curie transition temperature $\left(T_{\mathrm{C}}\right)$ of $\mathrm{CuCr}_{2} \mathrm{~S}_{4}, \mathrm{CuCr}_{2} \mathrm{Se}_{4}$ and $\mathrm{CuCr}_{2} \mathrm{Te}_{4}$ in the bulk are 377,430 and $360 \mathrm{~K}$, respectively. ${ }^{18,19}$ Band structure calculations for these compounds indicate a large density of states at the Fermi energy $\left(E_{\mathrm{F}}\right)$ in the majority spin channel as compared to the small density of states in the minority channel, 
suggesting metallic characteristics with a high degree of spin polarization. ${ }^{12,13,16,17}$

The exceptional spin-dependent properties along with ferromagnetic ordering at room temperature in bulk $\mathrm{CuCr}_{2} \mathrm{E}_{4}(\mathrm{E}=\mathrm{S}$, $\mathrm{Se}$ or $\mathrm{Te}$ ) compounds are attractive for practical applications. However, exploitation of their properties in nanodimensional form remains largely unexplored because of the lack of suitable synthetic methodologies for producing phase-pure material. Appropriate choice of molecular precursors and capping agents are essential ingredients for phase-pure synthesis of these complex chalcospinels in the form of nanocrystals. With careful choice of precursors, we have recently developed synthetic methodologies for $\mathrm{CuCr}_{2} \mathrm{E}_{4},\left(\mathrm{E}=\mathrm{S}\right.$, Se or Te) nanocrystals. ${ }^{20-24}$ Motivated by the semiconductor-like transport properties reported for bulk $\mathrm{CuCr}_{2} \mathrm{~S}_{4-x} \mathrm{Se}_{x}(0 \leq x \leq 4),{ }^{25}$ herein we report the colloidal synthesis of nanocrystals of $\mathrm{CuCr}_{2} \mathrm{~S}_{4-x} \mathrm{Se}_{x}(0 \leq x \leq 4)$ along with their detailed magnetic properties, complimented with electronic structure calculations. Our investigations reveal ferromagnetic ordering over the entire composition range, with a systematic increase in the superparamagnetic blocking and Curie temperatures with increasing Se content. Band structure calculations using density functional theory predict metallic characteristics in the bulk for all compositions, with a large difference in density of states in the majority and minority spin channels at the Fermi energy, suggesting high spin polarization. Furthermore, at reduced dimensions the calculations predict opening-up of a gap in minority spins at the Fermi level, rendering them closer to a true half-metallic system.

\section{Experimental details}

\section{Methods}

All chemicals were used as received and the solvents were dried over molecular sieves and purged with high purity argon for 30 minutes before use. 1-Dodecanethiol (1-DDT, 98.0\%), diphenyldiselenide (DPDS, 98.0\%) and copper chloride ( $\mathrm{CuCl}, 99.5 \%)$ were received from Alfa Aesar; chromium chloride $\left(\mathrm{CrCl}_{3} \cdot 6 \mathrm{H}_{2} \mathrm{O}\right.$, $\geq 99.0 \%$ ), oleylamine (OLA, 80-90\%) and octadecylamine (ODA, $\geq 99.0 \%$ ) were obtained from Acros Organics. Analytical grade hexane and ethanol were obtained from Aldrich Chemical Co.

\section{Synthesis of $\mathrm{CuCr}_{2} \mathrm{~S}_{4-x} \mathrm{Se}_{x}(0 \leq x \leq 4)$ nanocrystals}

Experiments were carried out in a fume hood under $\mathrm{N}_{2}$ atmosphere using a standard Schlenk line technique. For the synthesis of $\mathrm{CuCr}_{2} \mathrm{~S}_{4-x} \mathrm{Se}_{x}$ nanocrystals, $\mathrm{CuCl}(0.25 \mathrm{mmol}), \mathrm{CrCl}_{3} \cdot 6 \mathrm{H}_{2} \mathrm{O}$ $(0.50 \mathrm{mmol})$ and ODA $(10 \mathrm{gm})$ were degassed at room temperature for $30 \mathrm{~min}$ and put back under $\mathrm{N}_{2}$. This process was repeated three times and subsequently the reaction vessel was initially heated to $175-180{ }^{\circ} \mathrm{C}$ under vacuum followed by heating to $360-365{ }^{\circ} \mathrm{C}$ under nitrogen. Meanwhile, in a separate vessel a requisite amount of DPDS for achieving the desired mixed anion stoichiometry was dissolved in $2 \mathrm{~mL}$ of 1-DDT. This solution was then quickly introduced into the vessel containing the solution of metal salts when it reached $360{ }^{\circ} \mathrm{C}$. The reaction vessel cooled down during the injection process and was quickly reheated to $360{ }^{\circ} \mathrm{C}$ and maintained at this temperature for $1 \mathrm{~h}$ for the reaction to proceed. The mix was then cooled down to $65-70{ }^{\circ} \mathrm{C}$ and a mixture of hexane $(10 \mathrm{~mL})$ and ethanol $(15 \mathrm{~mL})$ was added to precipitate the product. The black precipitate was isolated via centrifugation. The precipitate was dispersed in hexane and reprecipitated by addition of ethanol. The above described method was also followed for the synthesis of $\mathrm{CuCr}_{2} \mathrm{Se}_{4}$ nanocrystal, but using an excess of diphenyldiselenide in OLA.

\section{Characterization and measurements}

Transmission electron microscopy (TEM) analysis was performed using a FEI-Tecnai, $200 \mathrm{kV}$ transmission electron microscope equipped with a CCD camera for STEM, HAADF detector, and EDX. TEM image non-linear processing was carried out using Gatan digital micrograph version 3.4. Powder X-ray diffraction (XRD) patterns were recorded on a Rigaku Smart Lab II instrument equipped with $\mathrm{Cu} \mathrm{K} \alpha$ radiation source operated at $40 \mathrm{kV}$ and 44 mA. PDXL v2.0 software package from Rigaku was used to process the powder XRD data. Scanning electron microscope (SEM) imaging and EDX mapping analysis were carried out using a JEOL 7000 FE SEM equipped with energy dispersive X-ray spectroscopy (EDX), wavelength dispersive X-ray spectroscopy (WDS), electron backscatter diffraction (EBSD), secondary electron (SE), backscattered electron (BE) and transmission electron (TE) detectors. Scanning transmission electron microscopy (STEM) high-angle annular dark field imaging and EDX elemental mapping were performed using a FEI Company Titan G2 80-200 TEM/STEM operated at $200 \mathrm{kV}$ and equipped with the X-FEG (an ultra-stable high-brightness Schottky FEG source), Super-X EDX detector system (4 windowless silicon drift detectors with a combined solid angle of $0.7 \mathrm{sr}$ ) and a spherical aberration corrector (CEOS DCOR) on the probe-forming optics. TEM image non-linear processing - Fourier filtering - was carried out using Gatan Digital Micrograph version 3.4. The Diamond $3.2 \mathrm{k}$ crystal viewer software was used to produce the simulated XRD patterns for all the NC compositions. Additionally, Rietveld refinement was performed using Match! software to match the experimental XRD patterns for the end members. Magnetic measurements were performed using a Quantum Design Dynacool Vibrating Sample Magnetometer (VSM) with MultiVu application.

\section{Band structure calculations}

The electronic structures of $\mathrm{CuCr}_{2} \mathrm{~S}_{4-x} \mathrm{Se}_{x}(0 \leq x \leq 4)$ were calculated within the PBE generalized gradient approximation ${ }^{26}$ to density functional theory, using the rotationally invariant " $+U$ " formalism of Liechtenstein et $a l^{27}$ All calculations were performed using the VASP ${ }^{28-31}$ code using PAW $^{32}$ pseudopotentials generated by Kresse and Joubert ${ }^{33}$ and a plane-wave cutoff of $368.6 \mathrm{eV}$. In the bulk calculations, we used the primitive 14-atom unit cell of the $F d \overline{3} m$ spinel lattice with a $16 \times 16 \times 16$ $\Gamma$-centered $k$-point mesh integrated using the tetrahedron method with Blöchl corrections. The computed nanostructures were constructed using the full 56-atom cubic cell with $15 \AA$ of vacuum on all sides. Our $\mathrm{PBE}+U$ calculations are conducted 
using the fully local double-counting correction with $U=3.4 \mathrm{eV}$ and $J=0.9 \mathrm{eV}$ for the $\mathrm{Cr} 3 \mathrm{~d}$ orbitals. For $\mathrm{CuCr}_{2} \mathrm{~S}_{4}$, we use a $\mathrm{PBE}+U$-relaxed structure with $a=9.81 \AA$ and the internal parameter $u=0.258$. The $\mathrm{PBE}+U$ relaxed lattice parameters for $\mathrm{CuCr}_{2} \mathrm{Se}_{4}$ were $a=10.31 \AA$ and $u=0.255$, with intermediate $a$ and differing $u$ values for the mixed phases. We did not find significant effects arising from the specific configuration of $S$ and Se ions at a given stoichiometry.

\section{Results and discussion}

Phase-pure synthesis of $\mathrm{CuCr}_{2} \mathrm{~S}_{4-x} \mathrm{Se}_{x}(0 \leq x \leq 4)$ nanocrystals is non-trivial, as it requires a delicate balance of reaction conditions. Copper and chromium ions are known to exist in variable oxidation states and thereby different stoichiometry binary chalcogenides can be readily formed. Moreover, copper ions readily react with chalcogenide donating agents to form copper chalcogenide nanocrystals, but chromium is relatively inert and requires extreme conditions for reaction. For the successful synthesis of $\mathrm{CuCr}_{2} \mathrm{~S}_{4-x} \mathrm{Se}_{x}(0 \leq x \leq 4)$ nanocrystals, we developed a colloidal hot-injection method. In this synthesis method, we inject a mixture of DPDS and 1-DDT into a reaction vessel containing copper and chromium precursors along with octadecylamine as capping ligand at a fairly high temperature (360-365 $\left.{ }^{\circ} \mathrm{C}\right)$. DPDS and 1-DDT function as selenium and sulfonating agents, respectively. We previously reported on the phase-pure synthesis of chromium-based sulfospinel nanocrystals and found that it is essential to use 1-DDT as a sulfonating agent, otherwise only the binary sulfides of copper and chromium are obtained. ${ }^{20}$ Similarly, it is important to use an appropriate selenium precursor for the synthesis of $\mathrm{CuCr}_{2} \mathrm{~S}_{4-x} \mathrm{Se}_{x}(0 \leq x \leq 4)$ nanocrystals, particularly one that is miscible with the other precursors and also avoids formation of binary copper/chromium selenides and $\mathrm{CuCr}_{2} \mathrm{Se}_{4}$ nanocrystals. Considering these factors, we investigated several commonly used selenium donating agents, including trioctylphosphine selenium (TOP-Se), selenium in octadecene (ODE-Se), and diphenyldiselenide. Among them, only diphenyldiselenide is found to produce phase-pure $\mathrm{CuCr}_{2} \mathrm{~S}_{4-x} \mathrm{Se}_{x}(0 \leq x \leq 4)$ nanocrystals. Injection of a mixture of diphenyldiselenide and 1-dodecanethiol into the high temperature reaction mix likely produce selenolate and thiolate ions, which then form coordination complex(es) with copper and/or chromium ions. Further heating of these in situ formed complexes generate the reactive nuclei needed for the formation and growth of the nanocrystals. It is noteworthy that the reactions have to be carried out at 360-365 ${ }^{\circ} \mathrm{C}$, which is unusually high for the solution-phase synthesis of chalcogen-containing nanocrystals. Reactions at lower reaction temperatures $\left(<350{ }^{\circ} \mathrm{C}\right)$ yield a mixture of the chalcospinel and non-magnetic $\mathrm{CuCrS}_{2} / \mathrm{CuCrSe}_{2}$ phases.

We characterized the nanocrystals using powder X-ray diffraction to confirm their phase purity, crystallinity and mixed anion compositions. X-ray diffraction patterns in Fig. 1(a) show distinct diffraction peaks corresponding primarily to the (311), (222), (400), (511) and (440) planes of the cubic phase spinel compounds. The peaks positions for $\mathrm{CuCr}_{2} \mathrm{~S}_{4}$ and $\mathrm{CuCr}_{2} \mathrm{Se}_{4}$ nanocrystals are in accordance with the standard diffraction patterns (ICDD: 21-0287 and 81-1986). A systematic shift in the diffraction peak positions to lower angles is observed with increasing selenium content, indicating lattice expansion due to formation of $\mathrm{CuCr}_{2} \mathrm{~S}_{4-x} \mathrm{Se}_{x}(0 \leq x \leq 4)$ solid solution. A steady enhancement of the (222) peak intensity is also noted with increasing selenium content. The diffraction peaks for all the patterns can be indexed to phase-pure chalcospinel compositions, with absence of any binary sulfide/selenide and ternary $\mathrm{CuCrS}_{2} / \mathrm{CuCrSe}_{2}$ impurity phases (Fig. S1, ESI $\dagger$ ). The lattice parameter $(a)$ values estimated from Rietveld simulation (Fig. 1(d) and (e)), of the diffraction patterns are $9.947 \pm 0.016 \AA$ for $x=0$ and $10.377 \pm 0.012 \AA$ for $x=4$ (Table 1 ). It can be noted that the estimated lattice parameter for $\mathrm{CuCr}_{2} \mathrm{~S}_{4-x} \mathrm{Se}_{x}(x=4)$ is somewhat higher that of literature values (10.357 ̊: ICDD: 03-065-4391). Crystal defects such as dislocation defects are known to expand the crystalline lattice. From TEM images in Fig. 2 and $3(\mathrm{~d})$ it can be seen that the nanocrystals do have imperfections and the detailed analysis to understand the type of crystalline defects demands extensive microscopic investigation, which is beyond the scope of this manuscript. Vegard's plot of the lattice parameter shows a linear trend in lattice expansion with increasing selenium content, as seen in Fig. 1(c). The elemental composition of the nanocrystals has been determined using SEM-EDX on a large number of particles. Within the accuracy of the measurements $( \pm 5 \%)$, the ratio of $\mathrm{Cu}, \mathrm{Cr}$, $\mathrm{S}$ and Se for the different compositions are found to be close to the expected values. The elemental compositions are provided in Table 1. The representative SEM-EDX spectrum for $\mathrm{CuCr}_{2} \mathrm{~S}_{4-x} \mathrm{Se}_{x}$ $(x=2)$ nanocrystals is given in Fig. 2(a), which clearly indicates the presence of $\mathrm{Cu}, \mathrm{Cr}, \mathrm{S}$ and Se. Further, the distribution of each element in the individual nanocrystals $(x=0-4)$ has been analyzed using TEM-EDX elemental mapping and it confirms uniform distribution of the elements (Fig. S2, ESI $\dagger$ for $x=3$ ). In addition we have carried out EDX analysis on individual particles with different sizes and did not observe any appreciable variation in the elemental compositions (Fig. S3 and Table S1, $\mathrm{ESI} \dagger$ for $x=3$ ).

We obtained shape and size information on the $\mathrm{CuCr}_{2} \mathrm{~S}_{4-x} \mathrm{Se}_{x}$ $(0 \leq x \leq 4)$ nanocrystals from transmission electron microscope images. TEM images of the nanocrystals are shown in Fig. 2(b)-(e). Cube-like nanocrystals of the end members $\mathrm{CuCr}_{2} \mathrm{~S}_{4}$ and $\mathrm{CuCr}_{2} \mathrm{Se}_{4}$ are observed, while irregular to nearly pyramidshaped nanocrystals are seen for the mixed $\mathrm{CuCr}_{2} \mathrm{~S}_{4-x} \mathrm{Se}_{x}$ $(1<x<3)$ compositions. The lateral dimensions of the nanocrystals, as estimated from the average of a large number of particles, are $21.0 \pm 2.4 \mathrm{~nm}$ for $x=0,14.6 \pm 3 \mathrm{~nm}$ for $x=1$, $16.0 \pm 2.8 \mathrm{~nm}$ for $x=2,15.6 \pm 2.5 \mathrm{~nm}$ for $x=3$, and $12 \pm 1.4 \mathrm{~nm}$ for $x=4$. These particles sizes are larger than the crystallite sizes estimated from powder X-ray diffraction (Table 1), suggesting that the individual nanocrystals may not be entirely single crystalline. High resolution transmission electron microscope (HRTEM) images of the nanocrystals are shown in Fig. 3(a)-(d). These images indicate that the nanocrystals are highly crystalline and exhibit well-defined lattice fringes. The distance 


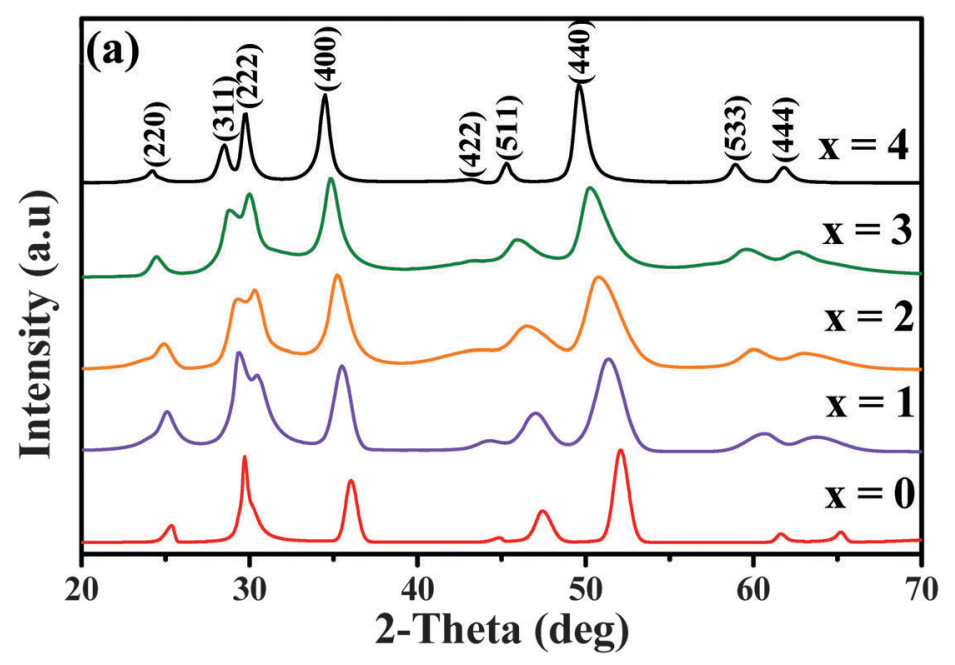

(b)

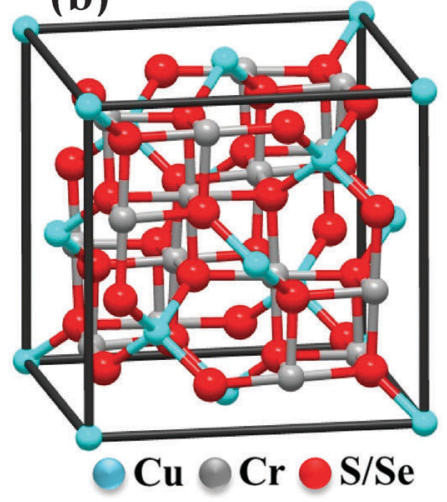

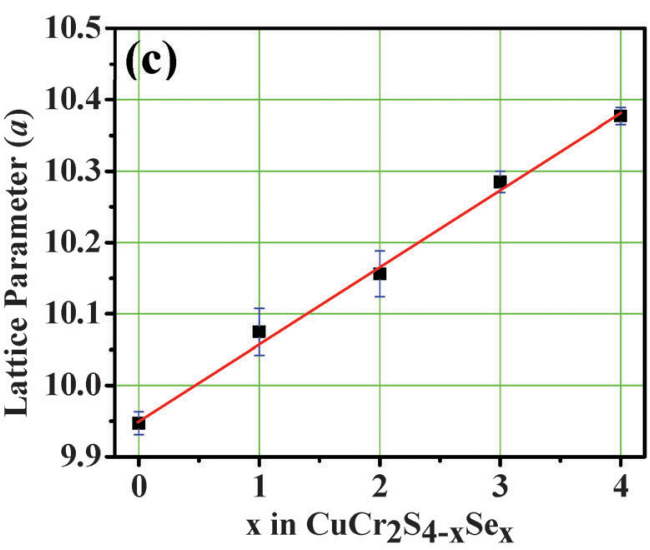
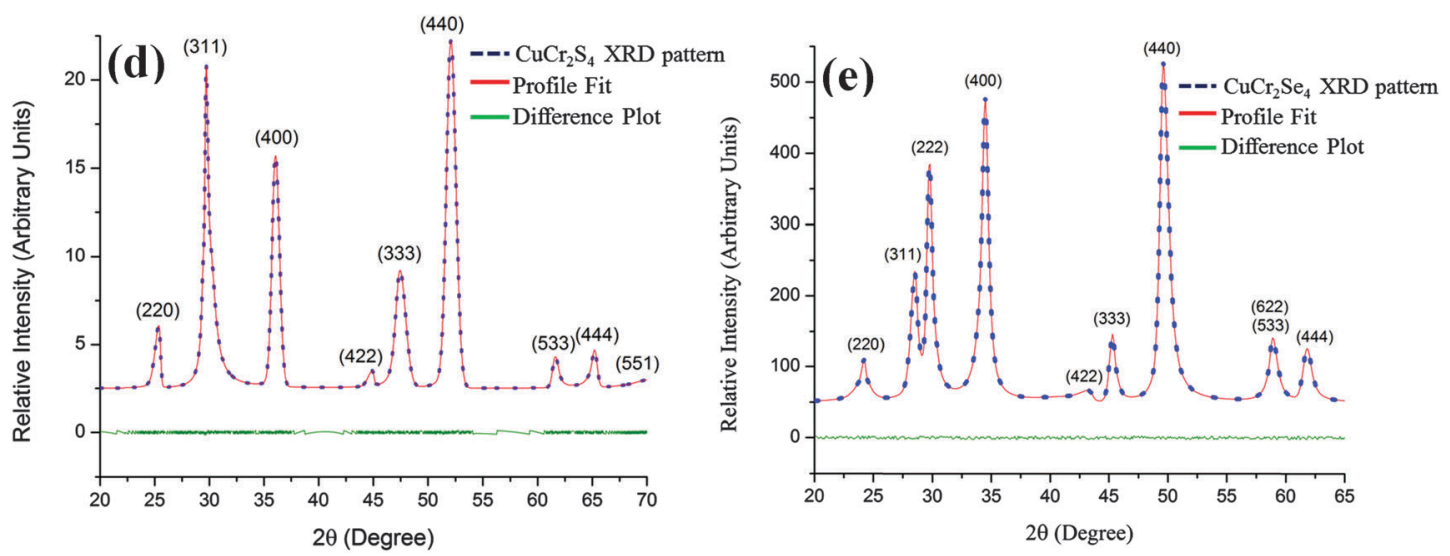

Fig. 1 (a) XRD patterns of $\mathrm{CuCr}_{2} \mathrm{~S}_{4-x} \mathrm{Se}_{x}(0 \leq x \leq 4)$ nanocrystals, (b) crystal structure of $\mathrm{CuCr}_{2} \mathrm{~S} / \mathrm{Se}_{4}$, (c) Vegard's plot. (d) and (e) Rietveld refinements showing good match with the experimental XRD data for $\mathrm{CuCr}_{2} \mathrm{~S}_{4}$ and $\mathrm{CuCr}_{2} \mathrm{Se}_{4}$ nanocrystals.

Table 1 EDX data, lattice constant, and particle size values from TEM images of $\mathrm{CuCr}_{2} \mathrm{~S}_{4-x} \mathrm{Se}_{x}(0 \leq x \leq 4)$ nanocrystals. The accuracy of the EDX data is estimated to be $\pm 5 \%$

\begin{tabular}{llclr}
\hline $\begin{array}{l}\mathrm{CuCr}_{2} \mathrm{~S}_{4-x} \mathrm{Se}_{x} \\
\text { nanocrystals }(x)\end{array}$ & $\begin{array}{l}\text { SEM-EDX data (\% at.) } \\
\mathrm{Cu}: \mathrm{Cr}: \mathrm{Se}: \mathrm{S}\end{array}$ & Lattice constant $(a)(\AA)$ & \multicolumn{1}{l}{$\begin{array}{l}\text { Ave. particle size } \\
(\mathrm{TEM})(\mathrm{nm})\end{array}$} & $\begin{array}{l}\text { Ave. crystallite size } \\
(\mathrm{XRD})(\mathrm{nm})\end{array}$ \\
\hline 0 & $12.8: 28.1: 0.0: 59.1$ & $9.947 \pm 0.016$ & $21.0 \pm 2.4$ & $11.0 \pm 0.8$ \\
1 & $12.6: 29.2: 13.9: 44.3$ & $10.075 \pm 0.033$ & $14.6 \pm 3.0$ & 1.0 \\
2 & $11.2: 28.3: 29.7: 30.8$ & $10.156 \pm 0.032$ & $16.0 \pm 2.8$ & $6.6 \pm 0.7$ \\
3 & $11.3: 26.7: 40.8: 21.2$ & $10.285 \pm 0.015$ & $15.6 \pm 2.5$ & $8.5 \pm 0.6$ \\
4 & $13.2: 28.3: 58.5: 0.0$ & $10.377 \pm 0.012$ & $12.0 \pm 1.4$ & $9.0 \pm 0.6$
\end{tabular}



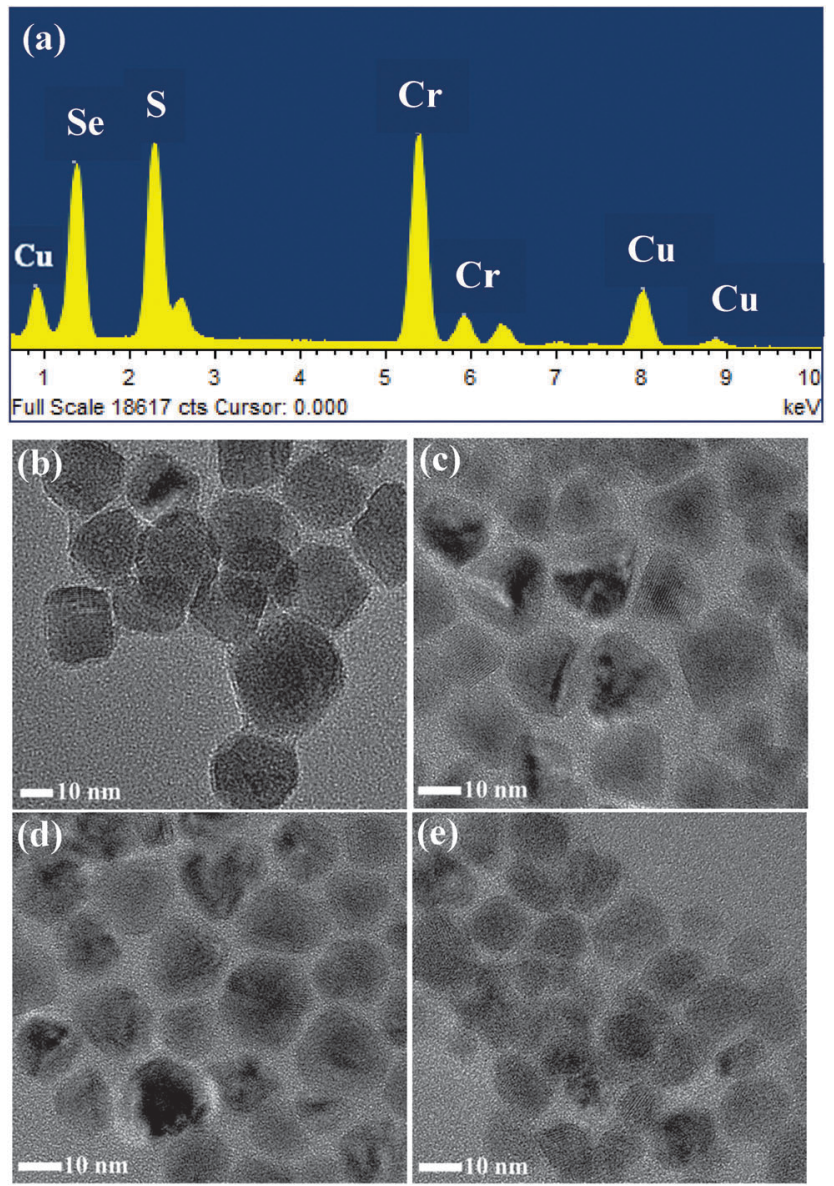

Fig. 2 (a) EDX spectrum of $\mathrm{CuCr}_{2} \mathrm{~S}_{4-x} \mathrm{Se}_{x}(x=2)$ nanocrystals. TEM images of $\mathrm{CuCr}_{2} \mathrm{~S}_{4-x} \mathrm{Se}_{x}$ nanocrystals (b) $x=0$, (c) $x=1$, (d) $x=2$, and (e) $x=4$
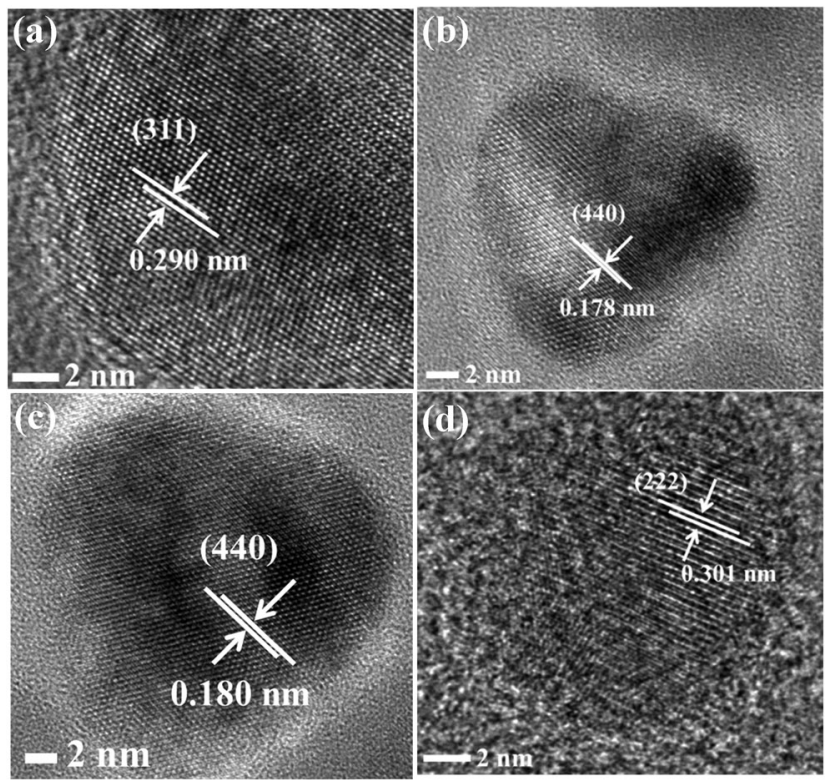

Fig. 3 HRTEM images of $\mathrm{CuCr}_{2} \mathrm{~S}_{4-x} \mathrm{Se}_{x}$ nanocrystals (a) $x=0$, (b) $x=1$, (c) $x=2$, and (d) $x=4$. between crystalline fringes for $\mathrm{CuCr}_{2} \mathrm{~S}_{4}$ nanocrystals is measured to be $0.29 \mathrm{~nm}$, corresponding to the (311) planes of cubic spinel. While the lattice fringe distances for $\mathrm{CuCr}_{2} \mathrm{~S}_{4-x} \mathrm{Se}_{x}(1<x<3)$ nanocrystals are between $0.178 \mathrm{~nm}$ and $0.182 \mathrm{~nm}$ for the (440) planes, indicating a systematic lattice expansion on substitution of sulfur with selenium. The $\mathrm{CuCr}_{2} \mathrm{Se}_{4}$ nanocrystals exhibit (222) crystal planes that are separated by $0.301 \mathrm{~nm}$. It is interesting to note that the morphology of $\mathrm{CuCr}_{2} \mathrm{~S}_{4-x} \mathrm{Se}_{x}(1<x<3)$ nanocrystals is different from that of the end members, which is also reflected in the crystalline lattice fringe distances as a consequence of different growth directions.

In order to investigate the magnetic properties of the nanocrystals such as saturation magnetization, superparamagnetic blocking temperature, and Curie temperature, we have carried out measurements using a variable temperature vibrating sample magnetometer (VSM). The effect of selenium substitution on the saturation magnetization and coercivity has been analyzed by measuring the magnetization $(M)$ as a function of applied magnetic field $(H)$ at 5 and $300 \mathrm{~K}$. The $M-H$ curves at $300 \mathrm{~K}$ for different composition $\mathrm{CuCr}_{2} \mathrm{~S}_{4-x} \mathrm{Se}_{x}(0 \leq x \leq 4)$ nanocrystals display superparamagnetic behavior with distinct " $\mathrm{S}$ " shape curves for the higher selenium content nanocrystals, as seen in Fig. S4 (ESI + ). However, measurements at $5 \mathrm{~K}$ (Fig. 4(a)) indicate ferromagnetic behavior for all compositions, with coercivity values ranging between 250 and 550 Oe, signifying soft-ferromagnetic

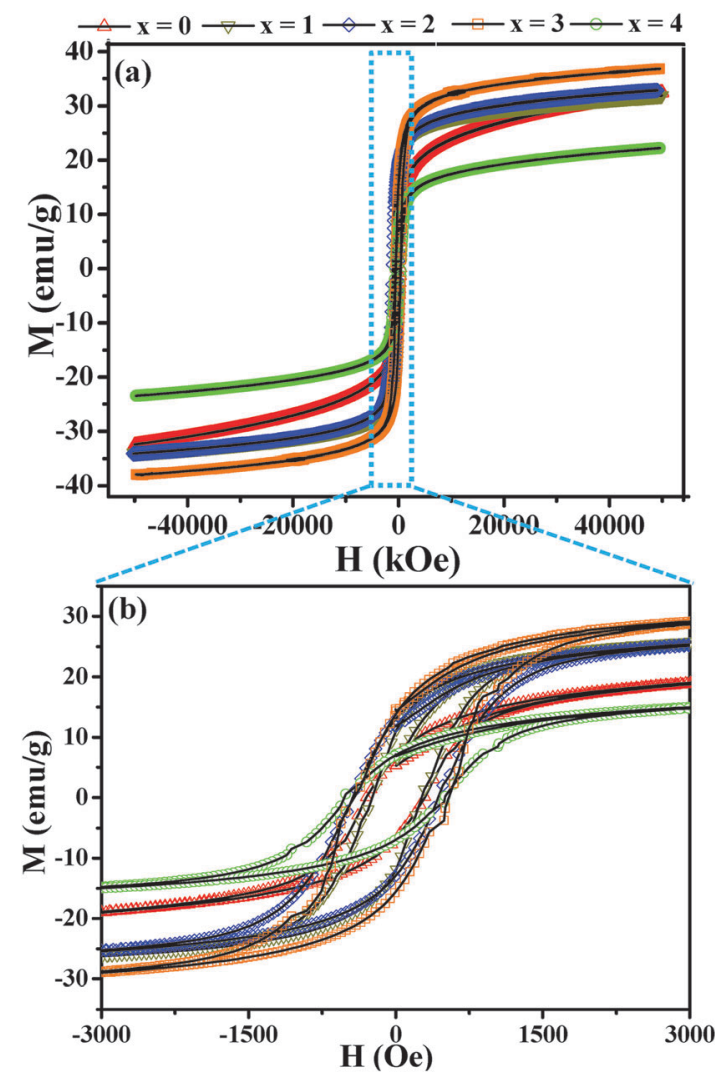

Fig. 4 (a) Magnetization (M) versus magnetic field $(H)$ measurements on $\mathrm{CuCr}_{2} \mathrm{~S}_{4-x} \mathrm{Se}_{x}(\mathrm{O} \leq x \leq 4)$ nanocrystals at $5 \mathrm{~K}$. (b) Expanded $M-H$ loops measured at $5 \mathrm{~K}$. 
characteristics (Fig. 4(b)). Additionally, the loops exhibit magnetization saturation at field values around 2 Tesla for all samples except for the $\mathrm{CuCr}_{2} \mathrm{~S}_{4}$ nanocrystals. The latter being in agreement with our previous observation..$^{20,34}$ The magnetization measured at 2 Tesla increases systematically from $32.0 \mathrm{emu} \mathrm{g}^{-1}$ for $x=0$ to $37.6 \mathrm{emu} \mathrm{g}^{-1}$ for $x=3$, followed by an unexpected drop in value for pure $\mathrm{CuCr}_{2} \mathrm{Se}_{4}$ nanocrystals. The magnetization for $\mathrm{CuCr}_{2} \mathrm{~S}_{4}$ nanocrystals is close to what we have reported earlier. ${ }^{20}$ However, the value for $\mathrm{CuCr}_{2} \mathrm{Se}_{4}$ nanocrystals is significantly lower than what we obtained for such nanocrystals synthesized using a different method. Notably, the average size of $\mathrm{CuCr}_{2} \mathrm{Se}_{4}$ nanocrystals is substantially smaller than the mixed composition nanocrystals. ${ }^{24,35}$ Materials at reduced dimensions can have more number of atoms at the surface than in the core, with the surface atoms having uncompensated dangling bonds due to inadequate passivation. The dangling bond spins are likely to couple antiferromagnetically with spins of the core atoms, leading to a reduced magnetization. Moreover, twin boundaries and point defects at cation and anion sites can contribute towards diluting the number of spins.

For determining the superparamagnetic blocking temperature of the different composition nanocrystals, we have performed field-cooled (FC) and zero-field-cooled (ZFC) measurements at different applied fields between 50 and 1000 Oe. In ZFC measurements, the spins are frozen in random orientations at a low temperature and by increasing the temperature in the presence of an applied field the spins are aligned along the field direction. However, at higher temperatures the thermal energy competes by aiding in flipping the spin direction. The temperature at which the spin disorientation occurs is referred to as the blocking temperature $\left(T_{\mathrm{B}}\right)$. The blocking temperature is determined by the magnetic anisotropy and volume of the material. From the coercivity values it can be noted that $\mathrm{CuCr}_{2} \mathrm{~S}_{4-x} \mathrm{Se}_{x}$ $(0 \leq x \leq 4)$ nanocrystals have low anisotropy and therefore their size has a significant influence on the blocking temperature. The ZFC curves are observed to be qualitatively similar for all the $\mathrm{CuCr}_{2} \mathrm{~S}_{4-x} \mathrm{Se}_{x}(0 \leq x \leq 4)$ nanocrystals.
Representative FC-ZFC plots for $\mathrm{CuCr}_{2} \mathrm{~S}_{4-x} \mathrm{Se}_{x}(x=3)$ nanocrystals are given in Fig. 5(a). In ZFC curve at 50 Oe field, a broad feature centered around $170 \mathrm{~K}$ can be observed, which is lower than the bifurcation temperature where the ZFC curve deviates from FC curve. With increasing applied field, the broad peak becomes more prominent and this peak along with the bifurcation point shift to lower temperatures. In an ideal case, nanocrystals with very narrow size dispersion show the peak and bifurcation point at the same temperature in FC-ZFC measurements, corresponding to the superparamagnetic blocking temperature $\left(T_{\mathrm{B}}\right)$. However, for our nanocrystals we observe the peak and bifurcation point at different temperatures, suggesting a fairly broad size distribution as confirmed from TEM images in Fig. 2. In this case, we can refer the peak to an ensemble average blocking temperature and the bifurcation point as the largest blocking temperature. Despite the size distribution, we observed a systematic shift in both temperatures to higher values with increasing selenium content in the nanocrystals. The largest nanocrystal blocking temperature for all compositions of $\mathrm{CuCr}_{2} \mathrm{~S}_{4-x} \mathrm{Se}_{x}(0 \leq x \leq 4)$ nanocrystals at different applied field values is plotted in Fig. 5(b).

Further, to study the effect of anion substitution on Curie transition temperature $\left(T_{\mathrm{C}}\right)$ in the $\mathrm{CuCr}_{2} \mathrm{~S}_{4-x} \mathrm{Se}_{x}(0 \leq x \leq 4)$ nanocrystals, we have carried out magnetization $(M)$ versus temperature $(T)$ measurements with an applied field of 2 Tesla. We observe an increase in the transition temperature with increasing selenium content (Fig. 6(a)). The temperature at which the magnetization value approaches zero are measured to be $340 \mathrm{~K}$ for $x=0 ; 320 \mathrm{~K}$ for $x=1 ; 350 \mathrm{~K}$ for $x=2 ; 370 \mathrm{~K}$ for $x=3$ and over $400 \mathrm{~K}$ for $x=4$ (Table 1 ). The values for $\mathrm{CuCr}_{2} \mathrm{~S}_{4}$ and $\mathrm{CuCr}_{2} \mathrm{Se}_{4}$ nanocrystals are in agreement with our previous reported results and are somewhat lower than the bulk $T_{\mathrm{C}}$ values. $^{20,24,25,36}$ Moreover, the observed lowering of Curie temperature $(320 \mathrm{~K})$ for $x=1$ and subsequent systematic increase of $T_{\mathrm{C}}$ for higher selenium content samples is similar to the trend reported for bulk $\mathrm{CuCr}_{2} \mathrm{~S}_{4-x} \mathrm{Se}_{x}(0 \leq x \leq 4) .{ }^{25}$ This behavior has been attributed to the existence of antiferron (disrupted ferromagnetic ordering)
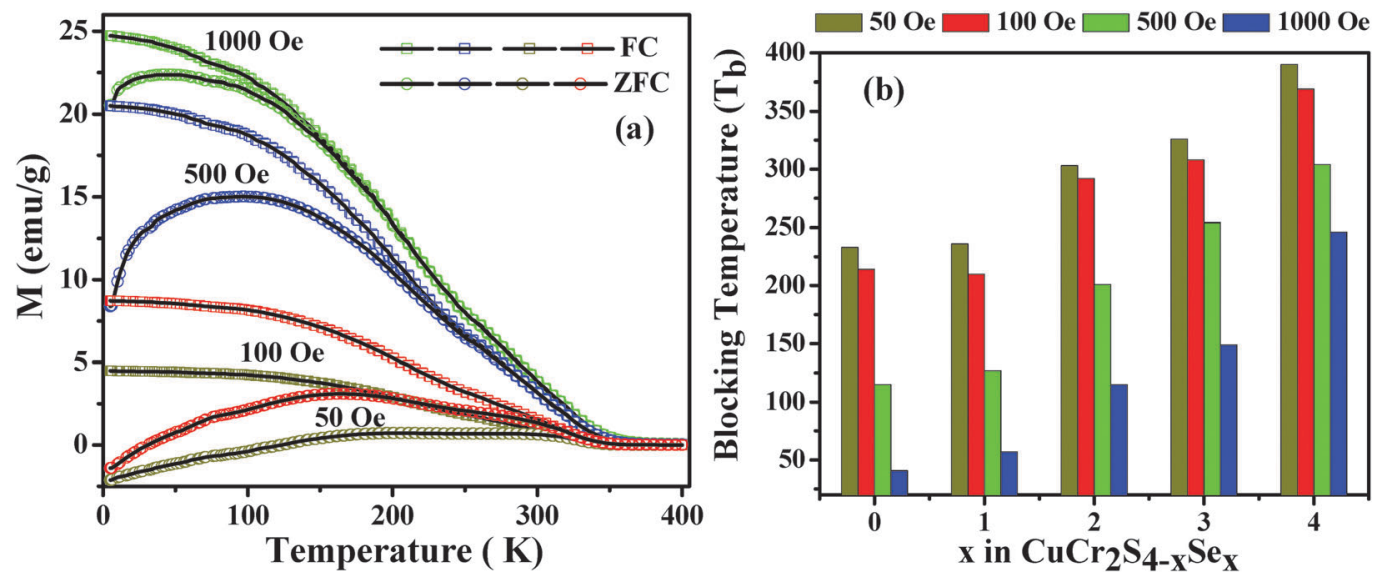

Fig. 5 (a) Magnetization (M) as a function of temperature for field-cooled (FC) and zero-field-cooled (ZFC) measurements at 1000 Oe, 500 Oe, 100 Oe and 50 Oe for $\mathrm{CuCr}_{2} \mathrm{~S}_{4-x} \mathrm{Se}_{x}(x=3)$. (b) Graph showing comparison of superparamagnetic blocking temperature $\left(T_{\mathrm{B}}\right)$ of $\mathrm{CuCr}_{2} \mathrm{~S}_{4-x} \mathrm{Se}_{x}(0 \leq x \leq 4)$ at different measurement fields. 

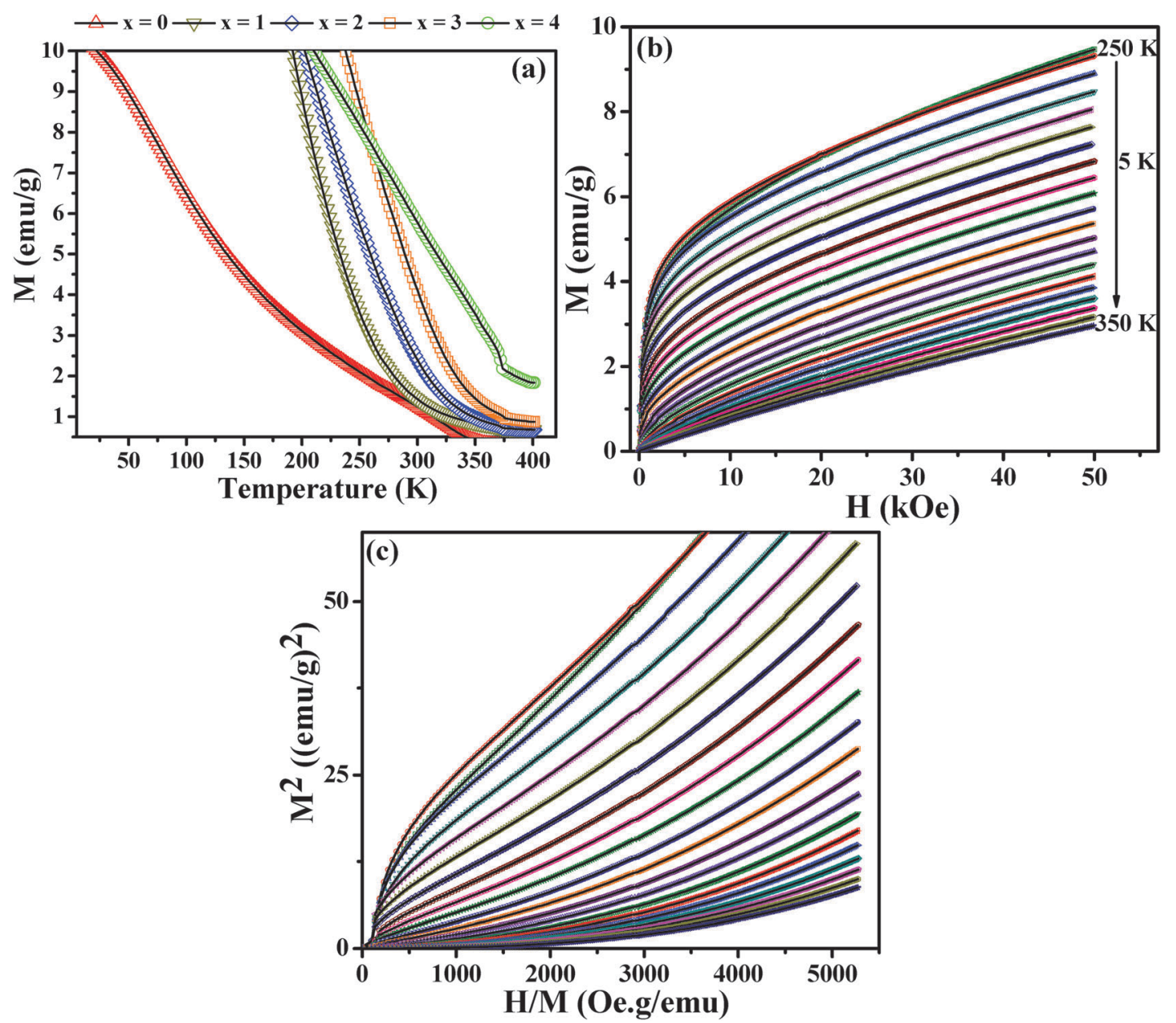

Fig. 6 (a) Magnetization (M) versus temperature ( $T)$ measurements on $\mathrm{CuCr}_{2} \mathrm{~S}_{4-x} \mathrm{Se}_{x}(0 \leq x \leq 4)$ nanocrystals with applied field of 20 kOe. Representative (b) isothermal and (c) Arrott plots for $\mathrm{CuCr}_{2} \mathrm{~S}_{4-x} \mathrm{Se}_{x}(x=2)$ nanocrystals.

regions in the mixed chalcospinels. ${ }^{25}$ We have also carried out isothermal measurements of the magnetization versus applied magnetic field for more accurate determination of the transition temperatures. Representative isothermal plot for $\mathrm{CuCr}_{2} \mathrm{~S}_{4-x} \mathrm{Se}_{x}$ $(x=2)$ is given in Fig. 6(b). Based on the isothermal measurements near the transition temperature, we have determined the $T_{\mathrm{C}}$ for the different composition samples by using the Arrott plot method (isothermal plots of $M^{2}$ vs. $\left.H / M\right),{ }^{37}$ as shown in Fig. 6(c). The Curie temperature determined using this method is in good agreement with those from $M-T$ measurements.

To help understand the variations in the magnetic properties with composition, we have carried out detailed band structure calculations both for the bulk and with reduced dimensions. We began by investigating the bulk properties of the $\mathrm{CuCr}_{2} \mathrm{~S}_{4-x} \mathrm{Se}_{x}$ $(0 \leq x \leq 4)$. We have previously published $\mathrm{PBE}+U$ calculations including $\mathrm{CuCr}_{2} \mathrm{~S}_{4}$ as the end case for $\mathrm{Co}_{x} \mathrm{Cu}_{1-x} \mathrm{Cr}_{2} \mathrm{~S}_{4} \cdot{ }^{22}$ We will not discuss the $x=0$ case in depth here but rather focus on how it differs from the substituted systems. Fig. 7 contains detailed views of the densities of states for the five systems in the vicinity of the Fermi level. Unsurprisingly, the plots are qualitatively quite similar due to the isovalent substitution, but we can observe several trends in the electronic structure as a function of Se content. We first consider the band gaps (always falling above the Fermi level, of course), noting that the majority and minority gaps both decrease with $x$ but at different rates. The majority gap separates hybridized p-d states from the empty Cr e $e_{g}$ states suggesting a charge transfer picture in which the size of the gap is primarily determined by an energy scale $\Delta$ that is related to the electronegativity of the anion (as given by the gap characterization picture of Zaanen, Sawatzky, and Allen ${ }^{38}$ ). Due to the empty minority $\mathrm{Cr}$ states, the energy difference between the occupied $t_{2 g}$ and unoccupied $e_{g}$ states will be given mostly by the crystal field splitting rather than the intraorbital $U$. This description is borne out by the gap trend: the majority gap decreases steadily with $x$, falling to $50 \%$ of the $\mathrm{CuCr}_{2} \mathrm{~S}_{4}$ value in $\mathrm{CuCr}_{2} \mathrm{Se}_{4}$.

The minority valence band is dominated by the anionic $\mathrm{p}$ states, while the states above the gap are $\mathrm{Cr} \mathrm{d}$, whose position is determined both by $U$ and the Hund's $J(3.4 \mathrm{eV}$ and $0.9 \mathrm{eV}$, respectively). This gap should be less affected by changes to the anion, and indeed the dependence of the size of the gap on $x$ is weaker than in the majority channel, particularly for $x \leq 2$. The difference in doping dependence (and in dependence on the strength of interactions in the B-site ion) gives one some control over not only the 


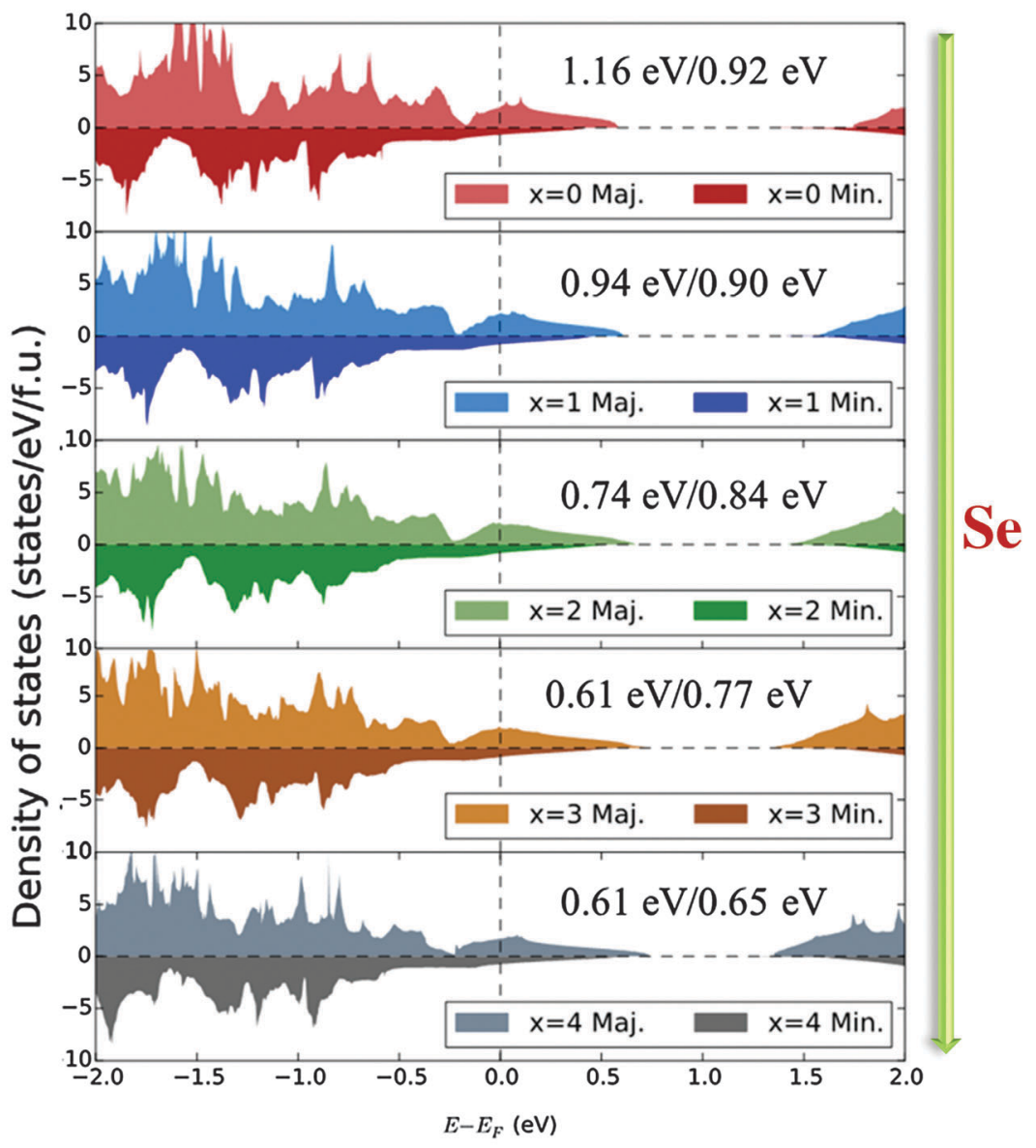

Fig. $7 \mathrm{GGA}+U$ densities of states for bulk $\mathrm{CuCr}_{2} \mathrm{~S}_{4-x} \mathrm{Se}_{x}$ with $x=0$ (top left) through $x=4$ (bottom). The size of the gap (above the Fermi level) and the spin polarization decrease with $x$, while the minority "gap" becomes larger than that in the majority channel above $x=1$.

absolute but the relative size of the gaps: the minority gap becomes the larger one between $x=1$ and $x=2$. This potential for tunability has been noted in the theoretical band structures of $\mathrm{CdCr}_{2} \mathrm{Te}_{4}{ }^{14}$ and the ferrites $\mathrm{NiFe}_{2} \mathrm{O}_{4}{ }^{39}$ and $\mathrm{CoFe}_{2} \mathrm{O}_{4}{ }^{40}$

Although the cell moment in our calculations follows the same trend as the magnetization in the nanocrystals - increasing through $x=3$ and then dropping somewhat in $\mathrm{CuCr}_{2} \mathrm{Se}_{4}$, we find that the magnetization decreases monotonically with $x$ from $100.6 \mathrm{emu} \mathrm{g}^{-1}$ at $x=0$ to $63.4 \mathrm{emu} \mathrm{g}^{-1}$ at $x=4$. We note that these are all much larger than the corresponding measurements, but as we discuss below, the computed magnetization does decrease in the finite systems.

We have estimated the Curie temperature by comparing the energy of ferromagnetic and antiferromagnetic $\mathrm{Cr}$ magnetic orientations and computing $T_{\mathrm{C}}$ within both the mean-field(MFA) and random-phase-approximations (RPA). Intra-B-site antiferromagnetism is necessarily frustrated (spinels typically exhibit antiferromagnetic order between the tetrahedrally coordinated A sites and the octahedrally coordinated B sites when both are magnetic), so we have chosen a reasonable configuration as our $\mathrm{AF}$ case. $T_{\mathrm{C}}$ is given by

$$
k_{\mathrm{B}} T_{\mathrm{C}}=\frac{2}{3} \sum_{i} J_{i}=\frac{1 M}{N_{q} 6} \sum_{q} E(q)
$$

in the MFA and by

$$
\frac{1}{k_{\mathrm{B}} T_{\mathrm{C}}}=\frac{16}{N_{q} M} \sum_{q} \frac{1}{E(q)}
$$

in the RPA, where we simply compare the $\mathrm{FM}(q=0)$ and $\mathrm{AF}(q=\pi)$ configurations. The latter expression will always give a lower (and more accurate) value than the mean-field one. The computed transition temperatures for the $x=0,2$ and 4 cases can be found in Table 2 (as explained above, the AF configuration is highly frustrated, and our method could not converge AF solutions in the larger supercells in the $x=1$ and 3 cases). We see the same general trend in $T_{\mathrm{C}}$ with $x$, including the non-monotonicity, and the RPA bulk values agree reasonably well with the experimental results in the finite nanocrystals. Our method for computing the exchange coupling is quite simple, and the results should not be over interpreted. However, it is clear that we can describe the magnetic structure of both the bulk and finite systems with some accuracy.

We have also investigated fully confined nanostructures, considering $1 \times 1 \times 1$ and $2 \times 2 \times 2$ nanocrystals (hereafter referred to as $1 \mathrm{~nm}$ and $2 \mathrm{~nm}$, respectively, roughly their length on each side) with $15 \AA$ of vacuum on all sides. In order to better understand the size dependence of the electronic and 
Table 2 Magnetic parameters of $\mathrm{CuCr}_{2} \mathrm{~S}_{4-x} \mathrm{Se}_{x}(0 \leq x \leq 4)$ nanocrystals; values deduced from $M-H, M-T$ measurements and compared with calculated and literature data. It was not possible to converge an antiferromagnetic solution in the larger supercells needed for the $x=1$ and $x=3$ systems, so we have only provided calculated Curie temperatures for the $x=0,2$, and 4 cases

\begin{tabular}{|c|c|c|c|c|c|c|}
\hline $\mathrm{CuCr}_{2} \mathrm{~S}_{4-r} \mathrm{Se}_{x}$ & \multirow{2}{*}{ 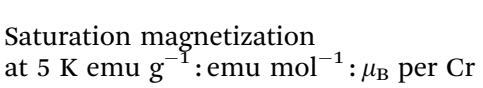 } & \multirow{2}{*}{$\begin{array}{l}\text { Coercivity } \\
\text { Oe at } 5 \mathrm{~K}\end{array}$} & \multicolumn{2}{|c|}{ Curie temp. $\left(T_{\mathrm{C}}\right) \mathrm{K}$} & \multicolumn{2}{|c|}{ Calculated Curie temp. $\left(T_{\mathrm{C}}\right) \mathrm{K}$} \\
\hline nanocrystals $(x)$ & & & Nanocrystals & Bulk $^{25}$ & MFA & RPA \\
\hline 0 & $32.0: 11503: 0.43$ & 300 & 340 & 377 & 606 & 404 \\
\hline 2 & $33.6: 13023: 0.58$ & 440 & 350 & 369 & 586 & 398 \\
\hline 3 & $37.6: 16287: 0.73$ & 440 & 370 & 392 & - & \\
\hline 4 & $23.0: 11012: 0.49$ & 540 & $>400$ & 437 & 656 & 450 \\
\hline
\end{tabular}

magnetic properties, we have also considered $2 \times 1 \times 1$ and $2 \times 2 \times 1$ systems. It is not possible to achieve fully symmetrical surface termination while simultaneously maintaining stoichiometry and avoiding disorder in the interior of the nanocrystals i.e. by only rearranging surface ions. For the $1 \mathrm{~nm}$ nanocrystals, we chose two terminations: one in which we did not alter the atomic positions from the bulk, yielding $3 \mathrm{Cu}$-terminated faces and $3 \mathrm{Cr}-\mathrm{S} / \mathrm{Se}$ terminated faces, and one in which we attempted to symmetrize the surfaces as much as possible, yielding 4 opposing Cu-terminated faces and 2 opposing $\mathrm{Cr}-\mathrm{S} / \mathrm{Se}$ terminated faces (Fig. 8(a) and (b)). We also examined two terminations for the $2 \mathrm{~nm}$ nanocrystal: the same bulk-like-terminated structure from the $1 \mathrm{~nm}$ system and a fully Cu-terminated structure that is nearly but not entirely symmetrical (Fig. 8(c) and (d)). We find in general that the more symmetrical configurations are lower in energy by close to $200 \mathrm{meV}$ per f.u.

We plot the density of states of the (symmetrically terminated) $x=0$ confined systems in Fig. 9. As we discuss below, only the $1 \mathrm{~nm}$ system is a true half metal, with a band gap of about $1 \mathrm{eV}$ in the minority channel; the majority valence band extends just beyond the Fermi level, yielding a highly spin-polarized metal.
In all four systems, surface $\mathrm{Cu} 4 \mathrm{~s}$ states join the $\mathrm{Cr} 3 \mathrm{~d}$ states at the bottom of the minority conduction band in the symmetric cell and break free of the conduction band to form localized gap states in the unaltered-bulk-position cell. This is common at surfaces: electrostatic repulsion is reduced at the vacuum interface, reducing the cost of occupying these states. For the same reason, in the occupied anionic p states the energy levels associated with chalcogen sites with broken bonds reduce their energy with respect to those with the full complement. This is likely the cause of the enhanced spin polarization in the finite clusters. We note that the more pronounced peak structure of the $1 \mathrm{~nm}$ system is quickly smeared out as we increase the system size and approach the bulk, and there are no qualitative differences in the electronic structure of the $2 \times 2 \times 1$ slab and the $2 \times 2 \times 2$ cubic particles.

The magnetic moment of the confined systems scales with size: for $x=0$, the $1 \mathrm{~nm}$ and $2 \mathrm{~nm}$ particles exhibit magnetizations of $86.2 \mathrm{emu} \mathrm{g}^{-1}$ and $94.1 \mathrm{emu} \mathrm{g}^{-1}$, respectively, the latter of which has already nearly recovered the bulk value. At $x=4$, the magnetization of the $1 \mathrm{~nm}$ system is $58.1 \mathrm{emu} \mathrm{g}^{-1}$, a similar reduction. Here, the decreased magnetization does not arise (a)

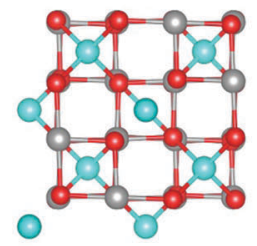

(c)
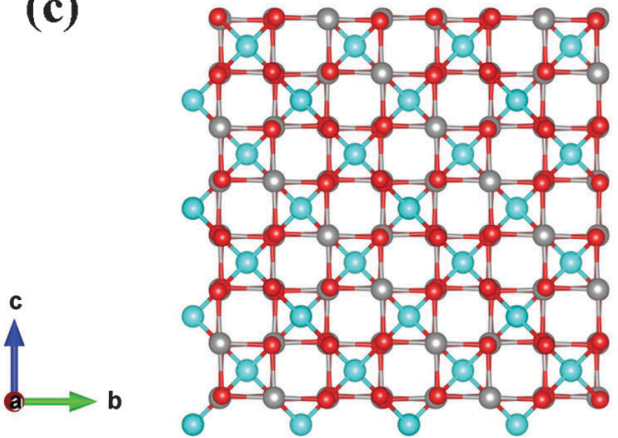

(b)

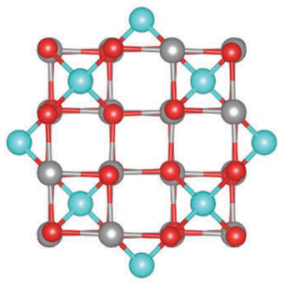

(d)



Fig. 8 Clusters used to model $1 \times 1 \times 1$ ( $a$ and $b$ ) and $2 \times 2 \times 2$ (c and d) nanocrystals in our PBE $+U$ calculations. We compared terminations based on the bulk structure ( $a$ and $c$ ) to those with more symmetrical Cu termination ( $b$ and d), finding the latter to be energetically favorable in all cases. 


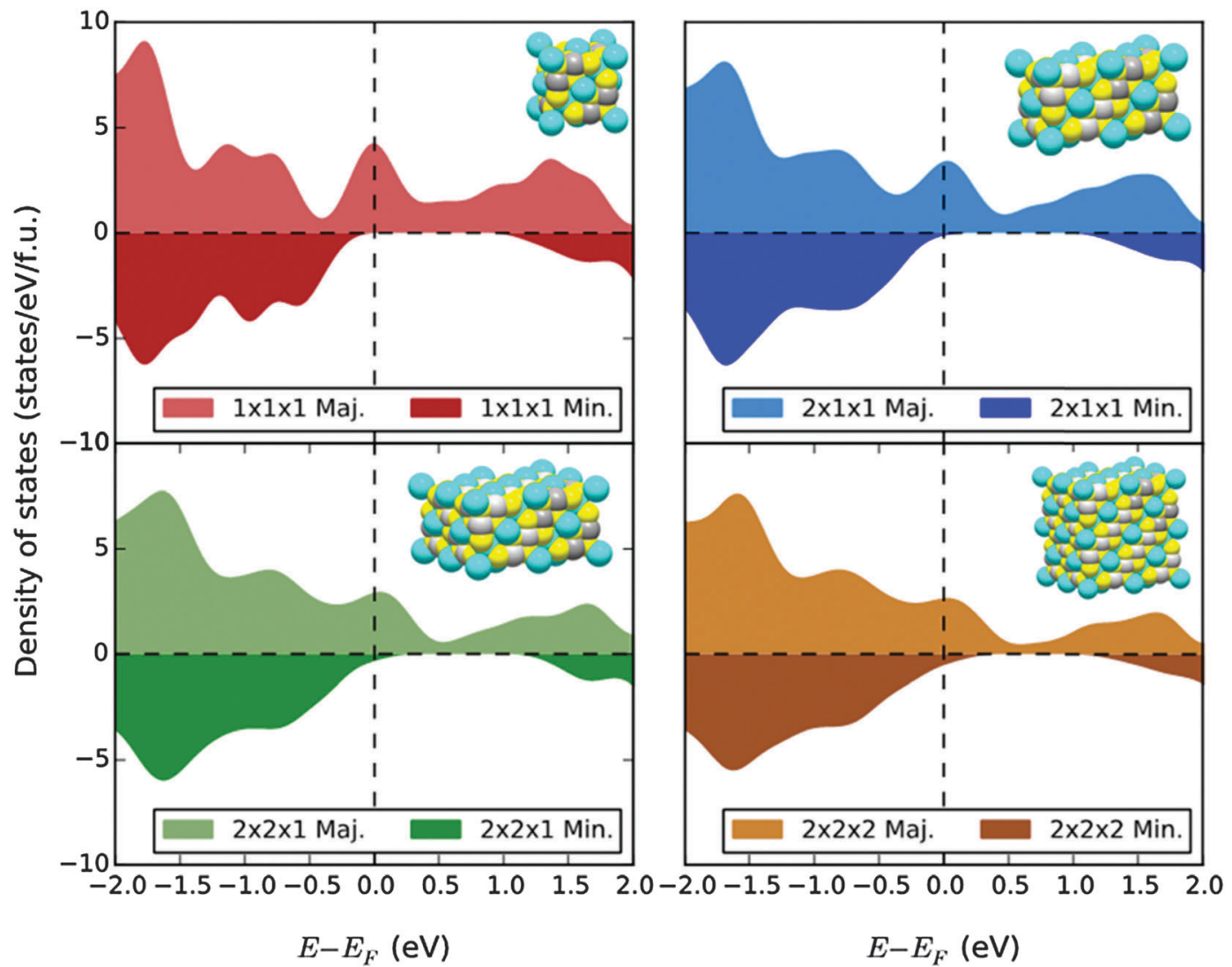

Fig. $9 \mathrm{GGA}+U$ densities of states for confined $\mathrm{CuCr}_{2} \mathrm{~S}_{4}$ nanostructures composed of 56 -atom cubic spinel unit cells. In all cases, the spin polarization is increased with respect to the bulk. The $1 \times 1 \times 1$ nanostructure (top left) becomes fully half-metallic, but all larger "particles" retain some minority states at the Fermi level. The $2 \times 2 \times 2$ system (bottom right) has nearly recovered the bulk electronic structure. (Inset: $\mathrm{Crystal}$ structures of $\mathrm{CuCr}_{2} \mathrm{~S}_{4}$ in corresponding size).

from a partially nonmagnetic surface ${ }^{23}$ but primarily from enhanced opposing moments induced throughout the $\mathrm{Cu}$ and $\mathrm{S} / \mathrm{Se}$ sites. We note that the more symmetrically terminated structures tend to have larger magnetizations.

We can define the spin polarization $P$ as

$$
P=\frac{n_{\uparrow}-n_{\downarrow}}{n_{\uparrow}+n_{\downarrow}}
$$

where $n_{\sigma}$ is the density of states of spin $\sigma$ at the Fermi energy. In Fig. 10, we plot the spin polarization as a function of system size in the confined structures (left) and of $x$ in the bulk (right). In the bulk systems, $P$ decreases with $x$, starting at about $50 \%$ in $\mathrm{CuCr}_{2} \mathrm{~S}_{4}$ and falling to $38 \%$ at $x=4$. However, the aforementioned electrostatic effects and reduced hybridization yield near-complete spin polarization (99\%) in the $1 \mathrm{~nm} x=0$ system. At $x=4, P$ falls to $89 \%$, still much larger than in the bulk system. In these confined structures, we note a dependence on the surface termination, particularly in the non-cubic cases. This is particularly pronounced in the $2 \times 1 \times 1$ rod, where the more symmetric termination gives a $10 \%$ increase in $P$ compared to the bulk termination. Polarization drops dramatically with system size, however. The $2 \mathrm{~nm}$ nanocrystal is only $66 \%$ polarized in the $x=0$ system (in both terminations). As a result, we suspect that nanocrystals of the sizes reported in this work would likely be nearly bulk-like in this regard.

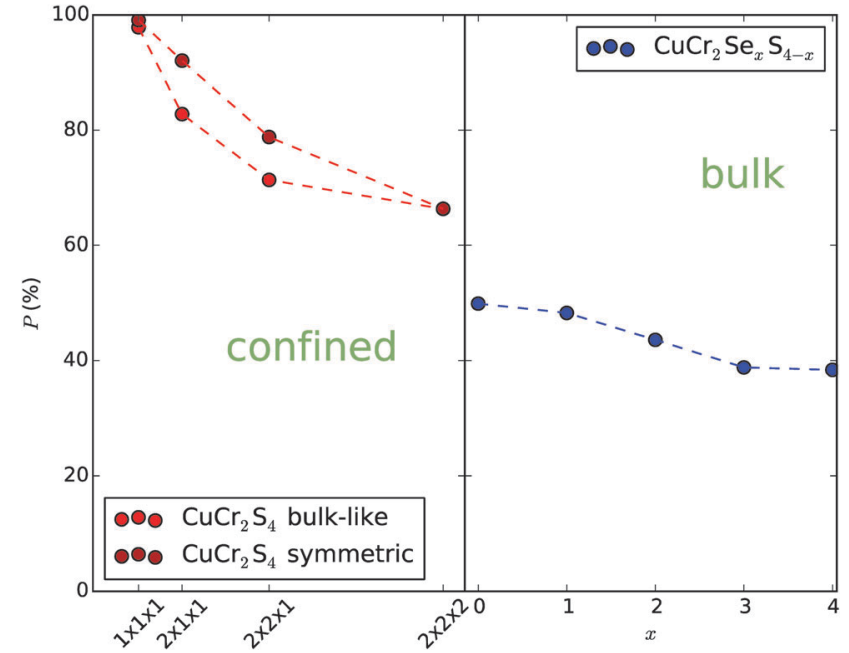

Fig. 10 Spin polarization from GGA+U calculations for confined $\mathrm{CuCr}_{2} \mathrm{~S}_{4}$ systems (as a function of number of cubic unit cells) and for bulk $\mathrm{CuCr}_{2} \mathrm{~S}_{4-x} \mathrm{Se}_{x}$ $(0 \leq x \leq 4)$ (as a function of $x$ ). The surface termination does not affect the polarization of the cubic particles. However, in the rod-like and slab-like structures symmetric termination (which is the lower in energy) increases the polarization by about $10 \%$. In both cases, polarization decreases quickly with system size. 


\section{Conclusion}

Nanocrystals of $\mathrm{CuCr}_{2} \mathrm{~S}_{4-x} \mathrm{Se}_{x}(0 \leq x \leq 4)$ have been synthesized over the entire composition using a mixture of 1-dodecanethiol and diphenyldiselenide as chalcogen agents and chlorides of copper and chromium as metal precursors. Systematic changes in the lattice parameter and elemental composition confirm formation of $\mathrm{CuCr}_{2} \mathrm{~S}_{4-x} \mathrm{Se}_{x}(0 \leq x \leq 4)$ nanocrystals. Depending on selenium content, the nanocrystals vary in size from $12 \pm 1.4 \mathrm{~nm}$ to $21 \pm 1.4 \mathrm{~nm}$. The magnetic transition temperature of the nanocrystals increases with increasing selenium content. Similarly, the saturation magnetization and coercivity values measured at $5 \mathrm{~K}$ are found to steadily increase up to $x=3$. Band structure calculations using density functional theory suggest ferromagnetic ordering over the entire composition range with calculated magnetization values and Curie transitions temperatures trend consistent with the experimental results. The spin polarization calculated for bulk $\mathrm{CuCr}_{2} \mathrm{~S}_{4-x} \mathrm{Se}_{x}(0 \leq x \leq 4)$ decreases from $50 \%$ to $38 \%$ with increasing selenium content. Interestingly, the calculation predicts a systematic increase in polarization value for the nanocrystals, with complete openingup of a gap at the Fermi level in the minority spin channel at reduced dimensions.

\section{Acknowledgements}

Synthesis, X-ray, TEM, SEM and magnetic characterization work was done at the University of Alabama, supported by the National Science Foundation under Grant No. CHE-1508259. Some TEM analysis was performed at the Center for Integrated Nanotechnologies, an Office of Science User Facility operated for the U.S. Department of Energy (DOE) Office of Science. Los Alamos National Laboratory, an affirmative action equal opportunity employer, is operated by Los Alamos National Security, LLC, for the National Nuclear Security Administration of the U.S. Department of Energy under contract DE-AC5206NA25396.

\section{References}

1 S. A. Wolf, D. D. Awschalom, R. A. Buhrman, J. M. Daughton, S. von Molnár, M. L. Roukes, A. Y. Chtchelkanova and D. M. Treger, Science, 2001, 294, 1488-1495.

2 H. C. Koo, J. H. Kwon, J. Eom, J. Chang, S. H. Han and M. Johnson, Science, 2009, 325, 1515-1518.

3 I. Žutić, J. Fabian and S. Das Sarma, Rev. Mod. Phys., 2004, 76, 323-410.

4 J. S. Moodera, L. R. Kinder, T. M. Wong and R. Meservey, Phys. Rev. Lett., 1995, 74, 3273-3276.

5 R. A. De Groot, F. M. Mueller, P. G. Van Engen and K. H. J. Buschow, Phys. Rev. Lett., 1983, 50, 2024-2027.

6 J. H. Park, E. Vescovo, H. J. Kim, C. Kwon, R. Ramesh and T. Venkatesan, Nature, 1998, 392, 794-796.

7 M. Jourdan, J. Minár, J. Braun, A. Kronenberg, S. Chadov, B. Balke, A. Gloskovskii, M. Kolbe, H. J. Elmers, G. Schönhense, H. Ebert, C. Felser and M. Kläui, Nat. Commun., 2014, 5, 3974.
8 Y. Guo, F. R. Shen and X. Y. Chen, Appl. Phys. Lett., 2012, 101, 012410.

9 C. P. Sun, C. L. Huang, C. C. Lin, J. L. Her, C. J. Ho, J. Y. Lin, H. Berger and H. D. Yang, Appl. Phys. Lett., 2010, 96, 2010-2012.

10 G. Catalan and J. F. Scott, Nature, 2007, 434, 364-367.

11 J. Bertinshaw, C. Ulrich, A. Günther, F. Schrettle, M. Wohlauer, S. Krohns, M. Reehuis, A. J. Studer, M. Avdeev, D. V. Quach, J. R. Groza, V. Tsurkan, A. Loidl and J. Deisenhofer, Sci. Rep., 2014, 4, 6079.

12 M. Park, S. Kwon, S. Youn and B. Min, Phys. Rev. B: Condens. Matter Mater. Phys., 1999, 59, 10018-10024.

13 S. Bordács, I. Kézsmárki, K. Ohgushi and Y. Tokura, New J. Phys., 2010, 12, 053039.

14 H. Sims, K. Ramasamy, W. H. Butler and A. Gupta, Appl. Phys. Lett., 2013, 103, 192402.

15 K. G. Nikiforov, Prog. Cryst. Growth Charact. Mater., 1999, 39, 1-104.

16 Y. H. A. Wang, A. Gupta, M. Chshiev and W. H. Butler, Appl. Phys. Lett., 2008, 92, 173-176.

17 Y. H. A. Wang, A. Gupta, M. Chshiev and W. H. Butler, Appl. Phys. Lett., 2009, 94, 173-176.

18 P. K. Baltzer, H. W. Lehmann and M. Robbins, Phys. Rev. Lett., 1965, 15, 493.

19 J. B. Goodenough, Solid State Commun., 1967, 5, 577-580.

20 K. Ramasamy, D. Mazumdar, Z. Zhou, Y.-H. A. Wang and A. Gupta, J. Am. Chem. Soc., 2011, 133, 20716-20719.

21 K. Ramasamy, D. Mazumdar, R. D. Bennett and A. Gupta, Chem. Commun., 2012, 48, 5656-5658.

22 K. Ramasamy, H. Sims, R. K. Gupta, D. Kumar, W. H. Butler and A. Gupta, Chem. Mater., 2013, 25, 4003-4009.

23 D. S. Negi, B. Loukya, K. Ramasamy, A. Gupta and R. Datta, Appl. Phys. Lett., 2015, 106, 182402.

24 Y. H. A Wang, N. Bao, L. Shen, P. Padhan and A. Gupta, J. Am. Chem. Soc., 2007, 129, 12408-12409.

25 L. I. Koroleva, J. Exp. Theor. Phys., 1994, 79, 153-162.

26 J. P. Perdew, K. Burke and M. Ernzerhof, Phys. Rev. Lett., 1996, 77, 3865.

27 A. I. Liechtenstein, V. I. Anisimov and J. Zaanen, Phys. Rev. B: Condens. Matter Mater. Phys., 1995, 52, R5467.

28 G. Kresse and J. Hafner, Phys. Rev. B: Condens. Matter Mater. Phys., 1993, 47, 558.

29 G. Kresse and J. Hafner, Phys. Rev. B: Condens. Matter Mater. Phys., 1994, 49, 14251.

30 G. Kresse and J. Furthmüller, Phys. Rev. B: Condens. Matter Mater. Phys., 1996, 54, 11169.

31 G. Kresse and J. Furthmüller, Comput. Mater. Sci., 1996, 6, 15.

32 P. E. Blöchl, Phys. Rev. B: Condens. Matter Mater. Phys., 1994, 50, 17953.

33 G. Kresse and D. Joubert, Phys. Rev. B: Condens. Matter Mater. Phys., 1999, 59, 1758.

34 A. I. Pankrats, A. M. Vorotynov, V. I. Tugarinov, S. M. Zharkov, D. A. Velikanov, G. M. Abramova, G. M. Zeer, 
K. Ramasamy and A. Gupta, J. Appl. Phys., 2014, 116, 054302.

35 C.-R. Lin, C.-L. Yeh, S.-Z. Lu, I. S. Lyubutin, S.-C. Wang and I. P. Suzdalev, Nanotechnology, 2010, 21, 235603.

36 I. Nakatani, H. Nose and K. Masumoto, J. Phys. Chem. Solids, 1978, 39, 743-749.

37 R. L. Hadimani, Y. Melikhov, J. E. Snyder and D. C. Jiles, J. Magn. Magn. Mater., 2008, 320, 696-698.
38 J. Zaanen, G. A. Sawatzky and J. W. Allen, Phys. Rev. Lett., 1985, 55, 418.

39 Q.-C. Sun, H. Sims, D. Mazumdar, J. X. Ma, B. S. Holinsworth, K. R. O'Neal, G. Kim, W. H. Butler, A. Gupta and J. L. Musfeldt, Phys. Rev. B: Condens. Matter Mater. Phys., 2012, 86, 205106.

40 B. S. Holinsworth, D. Mazumdar, H. Sims, Q.-C. Sun, M. K. Yurtisigi, S. K. Sarker, A. Gupta, W. H. Butler and J. L. Musfeldt, Appl. Phys. Lett., 2013, 103, 082406. 